\title{
ABCC11 - as key anti-odor target
}

\author{
T Baumann, Z Jovanovic, B Enthaler, T Schmidt-Rose, H Max, A Martin, Lara Terstegen, D Schweiger \\ From 1st International Workshop on Odor Spaces \\ Hannover, Germany. 4-7 September 2013
}

\section{Background}

In a previous publication, we showed that the $\mathrm{ABCC} 11$ transporter is crucial for the formation of the characteristic axillary odor and that a $538 \mathrm{G}>\mathrm{A}$ single nucleotide polymorphism (SNP) in the gene, prominent in Asians, leads to a nearly complete loss of the typical sweat odor components in these carriers. As it is unclear, whether ABCC11 is directly involved in the transport of these components, we performed $\mathrm{ABCC} 11$-mediated transporter experiments with various potential odor precursors and also studied possible pathways for the formation of these precursors.

\section{Methods}

Transport rates of various radioactive labeled peptide-conjugated potential precursors of 3-methyl-3-sulfanylhexanol (3M3SH) through membrane vesicles of insect cells overexpressing human $\mathrm{ABCC} 11$ were measured using a liquid scintillation counter. Control vesicles without ABCC11 were used to account for unspecific substrate transports. We analyzed relative expression levels of gglutamyl-transferase 1 (GGT1), a relevant key target for odor formation, and $\mathrm{ABCC} 11$ in different human tissues by quantitative real-time PCR using housekeeping genes for normalization. The localization of GGT1 and ABCC11 proteins in apocrine sweat glands were detected by immunohistochemistry of cryosections from axillary skin biopsies using polyclonal antibodies against the human proteins in combination with appropriate fluorescentlabeled secondary antibodies. For analyzing precursor metabolisms, MALDI-TOF-MS analyses were performed by use of an Autoflex III TOF mass spectrometer controlled by the flexControl 3.3 software package (Bruker Daltonics, Bremen, Germany).

\section{Results}

Whilst no ABCC11-mediated transport was detected for the dipeptide precursor Cys-Gly-3M3SH which can be found on the skin surface, the S-glutathione conjugate
SG-3M3SH was robustly taken up by ABCC11. These results illuminate the SG-3M3SH as a putative precursor of $3 \mathrm{M} 3 \mathrm{SH}$, a key determinant of axillary malodor, which then may undergo intravesicular maturation to generate Cys-Gly-3M3SH. GGT1 and ABCC11 transcripts were both present in axillary skin biopsies and curettage samples. In our immunohistological experiments, GGT1 signal was strongly detectable in the apical part of the secretory cells in the apocrine sweat glands and therefore in the same cellular region as ABCC11. As GGT1 is an enzyme which is known to catalyze the deglutamylation of glutathionyl-conjugates, we suggested that GGT1 is capable of transforming the SG-3M3SH to Cys-Gly-3M3SH. By incubating recombinant human GGT1 and SG-3M3SH and analyzing generated metabolites by MALDI-TOF-MS, we could prove the conversion of SG-3M3SH to Cys-Gly-3M3SH in vitro.

\section{Conclusion}

To sum up, we demonstrate that the functionality of $\mathrm{ABCC} 11$ is likely to play an important role in the generation of axillary malodor. Furthermore, we identify GGT1 as a key enzyme involved in the biosynthesis of Cys-Gly-3M3SH.

Published: 16 April 2014
doi:10.1186/2044-7248-3-S1-P16

Cite this article as: Baumann et al: ABCC11 - as key anti-odor target. Flavour 2014 3(Suppl 1):P16.

Beiersdorf AG, Research \& Development, Hamburg, Germany 